\title{
Optimal approximate solution theorems for Geraghty's proximal contractions in partially ordered sets via $\mathcal{w}$-distances
}

\author{
Chirasak Mongkolkeha ${ }^{\mathrm{a}}$, Eunyoung $\mathrm{Kim}^{\mathrm{b}}$, Yeol Je Cho $\mathrm{C}^{\mathrm{b}, \mathrm{c}, *}$ \\ ${ }^{a}$ Department of Mathematics, Statistics and Computer Sciences, Faculty of Liberal Arts and Science, Kasetsart University, \\ Kamphaeng-Saen Campus, Nakhonpathom 73140, Thailand. \\ ${ }^{b}$ Department of Mathematics Education and the RINS, Gyeongsang National University, Jinju 660-701, Korea. \\ ${ }^{c}$ Center for General Education, China Medical University, Taichung, 40402, Taiwan.
}

Communicated by Y. H. Yao

\begin{abstract}
The purpose of this paper is to solve some global optimization problems for Geraghty type proximal contractions in the setting of partially ordered sets with a metric by using a $w$-distance and an algorithm for determining such an optimal approximate solution, also, we give some examples to illustrate our main results. (C)2017 All rights reserved.
\end{abstract}

Keywords: Optimal approximate solution, best proximity point, Geraghty's proximal contraction, generalized distances, w-distance.

2010 MSC: 47H09, 47H10, 54H25.

\section{Introduction}

Several problems can be modeled as equations of the form $T x=x$, where $T$ is a given self-mapping defined on a subset of a metric space, a normed linear space, a topological vector space or some suitable spaces. However, if $T$ is a non-self-mapping from $A$ to $B$, then the aforementioned equation does not necessarily admit a solution. In this case, it is contemplated to find an approximate solution $x \in A$ such that the error $d(x, T x)$ is minimum, where $d$ is a distance function. In view of the fact that $d(x, T x)$ is at least $d(A, B)$, a best proximity point theorem guarantees the global minimization of $d(x, T x)$ by the requirement that an approximate solution $x$ satisfies the condition $d(x, T x)=d(A, B)$. Such optimal approximate solutions are called best proximity points of the mapping $\mathrm{T}$. In fact, best proximity theorems also serve as a natural generalization of fixed point theorems since a best proximity point serves as an optimal approximate solution to the equation $\mathrm{T} x=\mathrm{x}$.

A classical best approximation theorem was introduced by Fan [4], that is, if $A$ is a nonempty compact convex subset of a Hausdorff locally convex topological vector space $B$ and $T: A \rightarrow B$ is a continuous mapping, then there exists an element $x \in A$ such that $d(x, T x)=d(T x, A)$.

\footnotetext{
*Corresponding author

Email addresses: faascsm@ku.ac.th (Chirasak Mongkolkeha), eunyoung328@daum.net (Eunyoung Kim), yjcho@gnu.ac.kr (Yeol Je Cho)

doi:10.22436/jnsa.010.06.08
} 
Afterward, several authors, including Prolla [14], Reich [15], Sehgal and Singh [16, 17], have derived extensions of Fan's theorem in many directions. In 2012, Sadiq Basha [1] extended Banach's fixed point theorem for self-mappings to the setting of non-self-mappings which resolve global optimization problems via best proximity theorems in the setting of partially ordered sets equipped with a metric. For more results on the existence of best proximity points for some contractions, see [2, 3, 8-12, 19].

On the other hand, in 1996, Kada et al. [7] introduced a generalized metric, which is called the $w$-distance, and gave some examples of the $w$-distance and also proved Caristi's fixed point theorem, Ekeland's variational principle and the nonconvex minimization theorem (Takahashi [20]) by using the $w$-distance. Later, Shioji et al. [18] studied the relations between weakly contractive mappings and weakly Kannan mappings in metric spaces with the $w$-distance and the symmetric $w$-distance. Recently, Imdad and Rouzkard [6] proved some fixed point theorems for some contractions in complete metric spaces equipped with a partial order via the $w$-distance.

In this paper, we introduce the new classes of ordered proximal contractions, which are more generalized than the classes of ordered proximal contractions of first and second kinds by using the concept of Geraghty's function ([5]), and an algorithm for determining such an optimal approximate solution. Also, we prove some best proximity point theorems for Geraghty's proximal contractions type (A) and type (B) and the proposed algorithm in metric spaces with the $w$-distance. Furthermore, we give some examples to illustrate our main results.

\section{Preliminaries}

First, we give some definitions and their basic properties.

Definition 2.1. Let $(X, \preceq)$ be a partially ordered set. Two points $x, y \in X$ are said to be comparable with respect to $\preceq$ if either $x \preceq y$ or $y \preceq x$.

Definition 2.2. Let $\mathcal{S}$ be the family of functions from $[0, \infty)$ into $[0,1)$ satisfying the following condition:

$$
\beta\left(t_{n}\right) \rightarrow 1 \Longrightarrow t_{n} \rightarrow 0 .
$$

The function $\beta \in \mathcal{S}$ is called a Geraghty's function.

Example 2.3. For each $i \in\{1,2,3\}$, let $\beta_{i}:[0, \infty) \rightarrow[0,1)$ be the function defined by

(1) $\beta_{1}(t)= \begin{cases}\frac{\ln (t+1)}{t}, & \text { if } t>0 ， \\ 0, & \text { if } t=0 ｝\end{cases}$

(2) $\beta_{2}(t)= \begin{cases}\frac{e^{-t}}{t+1}, & \text { if } t>0, \\ k, & \text { if } t=0,0 \leqslant k<1 \text {; }\end{cases}$

(3) $\beta_{3}(t)= \begin{cases}\frac{1}{t+1}, & \text { if } t>0, \\ 0, & \text { if } t=0 .\end{cases}$

Then the function $\beta_{i} \in \mathcal{S}$ for each $i \in\{1,2,3\}$.

Definition 2.4. Let $(X, d)$ be a metric space. A function $p: X \times X \rightarrow[0, \infty)$ is called the $w$-distance on $X$ if the following conditions are satisfied:

(a) $p(x, z) \leqslant p(x, y)+p(y, z)$ for all $x, y, z \in X$;

(b) for any $x \in X, p(x, \cdot): X \rightarrow[0, \infty)$ is lower semi-continuous, i.e., if $x \in X$ and $y_{n} \rightarrow y \in X$, then

$$
p(x, y) \leqslant \liminf _{n \rightarrow \infty} p\left(x, y_{n}\right) ;
$$


(c) for any $\varepsilon>0$, there exists $\delta>0$ such that $p(z, x) \leqslant \delta$ and $p(z, y) \leqslant \delta$ imply $d(x, y) \leqslant \varepsilon$.

Let $X$ be a metric space with metric $d$. A $w$-distance $p$ on $X$ is said to be symmetric if $p(x, y)=p(y, x)$ for all $x, y \in X$. Obviously, every metric is a $w$-distance, but not conversely.

Next, we recall some examples in [21] to show that the $w$-distance is a generalized metric.

Example 2.5. Let $(X, d)$ be a metric space. A function $p: X \times X \rightarrow[0, \infty)$ defined by $p(x, y)=c$ for all $x, y \in X$ is a $w$-distance on $X$, where $c$ is a positive real number. But $p$ is not a metric since $p(x, x)=c \neq 0$ for any $x \in X$.

Example 2.6. Let $(X,\|\cdot\|)$ be a normed linear space. A function $p: X \times X \rightarrow[0, \infty)$ defined by $p(x, y)=$ $\|x\|+\|y\|$ for all $x, y \in X$ is a $w$-distance on $X$.

Example 2.7. Let $F$ be a bounded closed subset of a metric spaces $X$. Assume that $F$ contains at least two points and $c$ is a constant with $c \geqslant \delta(F)$, where $\delta(F)$ is the diameter of $F$. Then a function $p: X \times X \rightarrow[0, \infty)$ defined by

$$
p(x, y)= \begin{cases}d(x, y), & \text { if } x, y \in F, \\ c, & \text { if } x \notin F \text { or } y \notin F\end{cases}
$$

is a $w$-distance on $X$.

The following two lemmas are crucial for our results.

Lemma 2.8. $([7,21])$ Let $(X, d)$ be a metric space with the $w$-distance $p$. Let $\left\{x_{n}\right\}$ and $\left\{y_{n}\right\}$ be the sequences in $X$, where $\left\{\alpha_{n}\right\}$ and $\left\{\beta_{n}\right\}$ are the sequences in $[0, \infty)$ converging to zero. Then the following assertions hold: for all $x, y, z \in X$,

(1) If $p\left(x_{n}, y\right) \leqslant \alpha_{n}$ and $p\left(x_{n}, z\right) \leqslant \beta_{n}$ for any $n \in N$, then $y=z$. In particular, if $p(x, y)=0$ and $p(x, z)=0$, then $y=z$;

(2) If $\mathrm{p}\left(\mathrm{x}_{\mathrm{n}}, \mathrm{y}_{\mathrm{n}}\right) \leqslant \alpha_{\mathrm{n}}$ and $\mathrm{p}\left(\mathrm{x}_{\mathrm{n}}, z\right) \leqslant \beta_{\mathrm{n}}$ for any $\mathrm{n} \in \mathrm{N}$, then $\left\{\mathrm{y}_{\mathrm{n}}\right\}$ converges to $z$;

(3) If $\mathrm{p}\left(\mathrm{x}_{\mathrm{n}}, \mathrm{x}_{\mathrm{m}}\right) \leqslant \alpha_{\mathrm{n}}$ for any $\mathrm{n}, \mathrm{m} \in \mathrm{N}$ with $\mathrm{m}>\mathrm{n}$, then $\left\{\mathrm{x}_{\mathrm{n}}\right\}$ is a Cauchy sequence;

(4) If $\mathrm{p}\left(\mathrm{y}, \mathrm{x}_{\mathrm{n}}\right) \leqslant \alpha_{\mathrm{n}}$ for any $\mathrm{n} \in \mathrm{N}$, then $\left\{\mathrm{x}_{\mathrm{n}}\right\}$ is a Cauchy sequence.

Lemma 2.9 ([7]). Let $(X, d)$ be a metric space with the $w$-distance $p$. Let $\left\{x_{n}\right\}$ be a sequence in $X$ such that, for each $\varepsilon>0$, there exists $\mathrm{N}_{\varepsilon} \in \mathbb{N}$ such that $\mathrm{m}>\mathrm{n}>\mathrm{N}_{\varepsilon}$ implies $\mathrm{p}\left(\mathrm{x}_{\mathrm{n}}, \mathrm{x}_{\mathrm{m}}\right)<\varepsilon$, i.e., $\left(\lim _{\mathrm{m}, \mathrm{n} \rightarrow \infty} \mathrm{p}\left(\mathrm{x}_{\mathrm{n}}, \mathrm{x}_{\mathrm{m}}\right)=0\right)$. Then $\left\{x_{n}\right\}$ is a Cauchy sequence.

Let $X$ be a nonempty set such that $(X, \preceq)$ is a partially ordered set and $(X, d)$ is a complete metric space equipped with the $w$-distance $p$. For any nonempty subsets $A$ and $B$ of a metric space $X$, we give the following notations which are used in what follows:

$$
\begin{aligned}
p(A, B) & :=\inf \{p(x, y): x \in A \text { and } y \in B\} ; \\
A_{0} & :=\{x \in A: p(x, y)=p(A, B) \text { for some } y \in B\} ; \\
B_{0} & :=\{y \in B: p(x, y)=p(A, B) \text { for some } x \in A\} .
\end{aligned}
$$

Definition 2.10. A point $x \in A$ is called a best proximity point of a mapping $S: A \rightarrow B$ if it satisfies the following condition:

$$
p(x, S x)=p(A, B) .
$$

Definition 2.11. Let $S: A \rightarrow B$ be a mapping and $g: A \rightarrow A$ be an isometry. The mapping $S$ is said to preserve the isometric distance with respect to $\mathrm{g}$ if

$$
p(\operatorname{Sgx}, S g y)=p(S x, S y)
$$

for all $x, y \in A$. 
Definition 2.12. A mapping $S: A \rightarrow B$ is said to be proximally increasing if it satisfies the following condition:

$$
\left.\begin{array}{r}
x \preceq y, \\
p(u, S x)=d(A, B), \\
p(v, S y)=d(A, B),
\end{array}\right\} \Longrightarrow u \preceq v
$$

for all $u, v, x, y \in A$.

Definition 2.13. A mapping $S: A \rightarrow B$ is called an ordered Geraghty's proximal contraction type $(A)$ if there exists $\beta \in \mathcal{S}$ such that

$$
\left.\begin{array}{r}
x \preceq y, \\
p(u, S x)=p(A, B), \\
p(v, S y)=p(A, B),
\end{array}\right\} \Longrightarrow p(u, v) \leqslant \beta(p(x, y)) p(x, y)
$$

for all $u, v, x, y \in A$.

Definition 2.14. A mapping $\mathrm{T}: \mathrm{A} \rightarrow \mathrm{B}$ is called an ordered Geraghty's proximal contraction type (B) if there exists $\beta \in \mathcal{S}$ such that

$$
\left.\begin{array}{r}
x \preceq y, \\
p(u, S x)=p(A, B), \\
p(v, S y)=p(A, B),
\end{array}\right\} \Longrightarrow p(S u, S v) \leqslant \beta(p(S x, S y)) p(S x, S y)
$$

for all $u, v, x, y \in B$.

If $w=d$ and we take $\beta(t)=k$, where $k \in[0,1)$, then Geraghty's proximal contractions type $(A)$ and type (B) reduce to a proximal contraction of the first kind (Definition 2.15) and second kind (Definition 2.16), respectively, as follows:

Definition 2.15 ([1]). A mapping $S: A \rightarrow B$ is called a proximal contraction of the first kind if there exists $k \in[0,1)$ such that

$$
\left.\begin{array}{r}
x \preceq y, \\
d(u, S x)=d(A, B), \\
d(v, S y)=d(A, B),
\end{array}\right\} \Longrightarrow d(u, v) \leqslant k d(x, y)
$$

for all $u, v, x, y \in A$.

Definition 2.16 ([1]). A mapping $S: A \rightarrow B$ is called a proximal contraction of the second kind if there exists $k \in[0,1)$ such that

$$
\left.\begin{array}{r}
x \preceq y, \\
d(u, S x)=d(A, B), \\
d(v, S y)=d(A, B),
\end{array}\right\} \Longrightarrow d(S u, S v) \leqslant k d(S x, S y)
$$

for all $u, v, x, y \in A$.

\section{The main results}

In this section, we prove some global optimization theorems for Geraghty's proximal type contractions in the setting of partially ordered sets with a metric via the $w$-distance. Also, we give some examples to illustrate our main results. 
Theorem 3.1. Let $\mathrm{X}$ be a nonempty set such that $(\mathrm{X}, \preceq)$ is a partially ordered set and $(\mathrm{X}, \mathrm{d})$ is a complete metric space equipped with the $w$-distance $\mathrm{p}$. Let $\mathrm{A}$ and $\mathrm{B}$ be nonempty closed subsets of $\mathrm{X}$ such that $\mathrm{A}_{0}$ and $\mathrm{B}_{0}$ are nonempty. Assume that the mappings $\mathrm{S}: \mathrm{A} \rightarrow \mathrm{B}$ and $\mathrm{g}: \mathrm{A} \rightarrow \mathrm{A}$ such that $\mathrm{S}$ preserves isometric distance with respect to $\mathrm{g}$ and satisfy the following conditions:

(a) $S$ is a proximally increasing ordered Geraghty's proximal contractions type $(A)$ and type (B) with $S\left(A_{0}\right) \subseteq B_{0}$;

(b) $g$ is a surjective isometry and its inverse is an increasing mapping with $A_{0} \subseteq g\left(A_{0}\right)$;

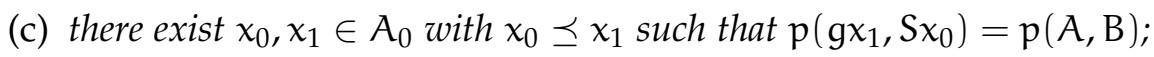

(d) if $\left\{x_{n}\right\}$ is an increasing sequence in $A$ converging to $x$, then $x_{n} \preceq x$ for all $n \geqslant 1$ and $\lim _{n \rightarrow \infty} p\left(x, x_{n}\right)=0$.

Then there exists a point $x \in A$ such that $p(g x, S x)=p(A, B)$. Moreover, for any $x_{0} \in A_{0}$, the sequence $\left\{x_{n}\right\}$ defined by $\mathrm{p}\left(\mathrm{gx_{n+1 }}, \mathrm{S} \mathrm{x}_{\mathrm{n}}\right)=\mathrm{p}(\mathrm{A}, \mathrm{B})$ converges to the point $\mathrm{x}$.

Proof. From the condition (c), there exist $x_{0}, x_{1} \in A_{0}$ such that $x_{0} \preceq x_{1}$ and

$$
p\left(g x_{1}, S x_{0}\right)=p(A, B) .
$$

Since $S\left(A_{0}\right) \subseteq B_{0}$ and $A_{0} \subseteq g\left(A_{0}\right)$, there exists $x_{2} \in A_{0}$ such that

$$
p\left(g x_{2}, S x_{1}\right)=p(A, B) .
$$

Since $S$ is proximally increasing, $g x_{1} \preceq g x_{2}$. Also, since the inverse of $g$ is an increasing mapping, $x_{1} \preceq x_{2}$. By the same method, we can find $x_{n}, x_{n+1} \in A_{0}$ such that $x_{n} \preceq x_{n+1}$ and

$$
p\left(g x_{n+1}, S x_{n}\right)=p(A, B) .
$$

Since $S\left(A_{0}\right) \subseteq B_{0}$ and $A_{0} \subseteq g\left(A_{0}\right), S$ is an ordered Geraghty's proximal contraction type $(A), g$ is an isometry and the property of $\beta$, it follows that, for each $n \geqslant 1$,

$$
p\left(x_{n}, x_{n+1}\right)=p\left(g x_{n}, g x_{n+1}\right) \leqslant \beta\left(p\left(x_{n-1}, x_{n}\right)\right) p\left(x_{n-1}, x_{n}\right) \leqslant p\left(x_{n-1}, x_{n}\right),
$$

which implies that the sequence $\left\{p\left(x_{n}, x_{n+1}\right)\right\}$ is non-increasing and converges to a number $r \geqslant 0$, i.e.,

$$
\lim _{n \rightarrow \infty} p\left(x_{n}, x_{n+1}\right)=r .
$$

Suppose that $r>0$. Then we have

$$
\frac{p\left(x_{n}, x_{n+1}\right)}{p\left(x_{n-1}, x_{n}\right)} \leqslant \beta\left(p\left(x_{n-1}, x_{n}\right)\right)
$$

which implies that $\lim _{n \rightarrow \infty} \beta\left(p\left(x_{n-1}, x_{n}\right)\right)=1$ and, since $\beta \in \mathcal{S}$, we obtain $r=0$. Therefore, it follows that

$$
\lim _{n \rightarrow \infty} p\left(x_{n}, x_{n+1}\right)=0
$$

Now, we claim that

$$
\lim _{n, m \rightarrow \infty} p\left(x_{m}, x_{n}\right)=0 .
$$

Suppose that there exist $\delta>0$ and two subsequences $\left\{x_{m_{k}}\right\},\left\{x_{n_{k}}\right\}$ of the sequence $\left\{x_{n}\right\}$ such that, for any $n_{k}>m_{k} \geqslant k$ with $n_{k}$ is the smallest number,

$$
p\left(x_{m_{k}}, x_{n_{k}}\right) \geqslant \delta, \quad p\left(x_{m_{k}}, x_{n_{k}-1}\right)<\delta
$$


for any $k \geqslant 1$. Then we have

$$
\delta \leqslant p\left(x_{m_{k}}, x_{n_{k}}\right) \leqslant p\left(x_{m_{k}}, x_{n_{k}-1}\right)+p\left(x_{n_{k}-1}, x_{n_{k}}\right) \leqslant \delta+p\left(x_{n_{k}-1}, x_{n_{k}}\right) .
$$

By, using (3.2) and (3.4), we have

$$
\lim _{k \rightarrow \infty} p\left(x_{m_{k}}, x_{n_{k}}\right)=\delta
$$

Next, we show that

$$
\lim _{k \rightarrow \infty} \sup p\left(x_{m_{k}+1}, x_{n_{k}+1}\right)<\delta .
$$

If $\lim _{k \rightarrow \infty} \sup p\left(x_{m_{k}+1}, x_{n_{k}+1}\right) \geqslant \delta$, then there exists $k_{r}$ such that

$$
\lim _{r \rightarrow \infty} p\left(x_{m_{k_{r}}+1}, x_{n_{k_{r}}+1}\right)=\varepsilon \geqslant \delta .
$$

By the definition of the sequence $\left\{x_{n}\right\}$, for any $n_{k_{r}}>m_{k_{r}} \geqslant k_{r} \geqslant r$, we can assume that $x_{m_{r}+1} \preceq x_{n_{r}+1}$ with

$$
p\left(g x_{n_{k_{r}}+1}, S x_{n_{k_{r}}}\right)=p(A, B), \quad p\left(g x_{m_{k_{r}}+1}, S x_{m_{k_{r}}}\right)=p(A, B) .
$$

Therefore,

$$
p\left(x_{m_{k r}+1}, x_{n_{k_{r}}+1}\right) \leqslant p\left(g x_{m_{k_{r}}+1}, g x_{n_{k_{r}}+1}\right) \leqslant \beta\left(p\left(x_{m_{k_{r}}}, x_{n_{k_{r}}}\right)\right) p\left(x_{m_{k_{r}}}, x_{n_{k_{r}}}\right) .
$$

It follows that

$$
1 \leqslant \frac{\varepsilon}{\delta}=\lim _{r \rightarrow \infty} \frac{p\left(x_{m_{k_{r}}+1}, x_{n_{k_{r}}+1}\right)}{p\left(x_{m_{k_{r}}}, x_{n_{k_{r}}}\right)} \leqslant \lim _{r \rightarrow \infty} \beta\left(p\left(x_{m_{k_{r}}}, x_{n_{k_{r}}}\right)\right) \leqslant 1
$$

Therefore, we have

$$
\lim _{k \rightarrow \infty} \beta\left(p\left(x_{m_{k_{r}}}, x_{n_{k_{r}}}\right)\right)=1 .
$$

Hence, by the property of $\beta \in \mathcal{S}$, we have $\delta=0$, which is a contradiction, and so (3.3) holds. By Lemma 2.9 , the sequence $\left\{x_{n}\right\}$ is a Cauchy sequence. Since $A$ is a closed subset of a complete metric space $X,\left\{x_{n}\right\}$ converges to a point $x \in A$ with $x_{n} \preceq x$. Consequently, it follows that $g x_{n} \rightarrow g x$ as $n \rightarrow \infty$. Further, since $S$ is an ordered Geraghty's proximal contraction type (B) and $S$ preserves the isometric distance with respect to $g$, we have

$$
p\left(S x_{n}, S x_{n+1}\right)=p\left(S g x_{n}, S g x_{n+1}\right) \leqslant \beta\left(p\left(S x_{n-1}, S x_{n}\right)\right) p\left(S x_{n-1}, S x_{n}\right) \leqslant p\left(S x_{n-1}, S x_{n}\right),
$$

which means that the sequence $\left\{p\left(S x_{n+1}, S x_{n}\right)\right\}$ is non-increasing and bounded below. By the same argument as above, we can see that

$$
\lim _{m, n \rightarrow \infty} p\left(S x_{m}, S x_{n}\right)=0
$$

and so $\left\{S x_{n}\right\}$ is a Cauchy sequence. Suppose that $\left\{S x_{n}\right\}$ converges to a point $y \in$ B. By (3.1), (3.5), assumption $(d)$, and the lower semi-continuity of $p\left(x_{n}, \cdot\right)$, we have

$$
\begin{aligned}
p(A, B) \leqslant p(g x, y) & \leqslant \liminf _{m, n \rightarrow \infty} p\left(g x, g x_{n+1}\right)+\liminf _{m, n \rightarrow \infty} p\left(g x_{n+1}, S x_{n}\right)+\liminf _{m, n \rightarrow \infty} p\left(S x_{n}, S x_{m}\right) \\
& =\lim _{n \rightarrow \infty} p\left(g x, g x_{n+1}\right)+p(A, B)+\lim _{m, n \rightarrow \infty} p\left(S x_{n}, S x_{m}\right) \\
& =p(A, B) .
\end{aligned}
$$


Therefore, $p(g x, y)=p(A, B)$ and hence $g x \in A_{0}$.

Next, we show that $x \in A_{0}$. Since $A_{0} \subseteq g\left(A_{0}\right)$, we have $g x=g z$ for some $z \in A_{0}$. By the isometry of $g$, (3.3), and the lower semi-continuity of $p\left(x_{n}, \cdot\right)$, we can see that

$$
p\left(x_{n}, z\right)=p\left(g x_{n}, g z\right)=p\left(g x_{n}, g x\right)=p\left(x_{n}, x\right) \leqslant \liminf _{m \rightarrow \infty} p\left(x_{n}, x_{m}\right) \leqslant \alpha_{n}
$$

for a sequence $\left\{\alpha_{n}\right\}$ converging to zero. Again, by (3.3) and the lower semi-continuity of $p\left(x_{n}, \cdot\right)$, we have

$$
p\left(x_{n}, x\right) \leqslant \liminf _{m \rightarrow \infty} p\left(x_{n}, x_{m}\right) \leqslant \beta_{n}
$$

where $\beta_{n}=\alpha_{n}$ for each $n \geqslant 1$. Using (3.6), (3.7), and Lemma 2.8, we obtain $x=z \in A_{0}$. In fact, $\mathrm{S}\left(\mathrm{A}_{0}\right) \subseteq \mathrm{B}_{0}$, there exists $\mathrm{u} \in \mathrm{A}$ such that

$$
p(u, S x)=p(A, B) .
$$

Since $S$ is an ordered Geraghty's proximal contraction type $(A)$, it follows from (3.1) and (3.8) that

$$
p\left(g x_{n+1}, u\right) \leqslant \beta\left(p\left(x_{n}, x\right)\right) p\left(x_{n}, x\right) \leqslant p\left(x_{n}, x\right)
$$

for each $n \geqslant 1$. Again, by (3.3) and the lower semi-continuity of $p\left(x_{n}, \cdot\right)$, we have

$$
p\left(g x_{n+1}, u\right) \leqslant p\left(x_{n}, x\right) \leqslant \liminf _{m \rightarrow \infty} p\left(x_{n}, x_{m}\right) \leqslant \alpha_{n}
$$

for a sequence $\left\{\alpha_{n}\right\}$ converging to zero. Further, it follows that

$$
p\left(g x_{n+1}, g x\right)=p\left(x_{n+1}, x\right) \leqslant \liminf _{m \rightarrow \infty} p\left(x_{n+1}, x_{m}\right) \leqslant \xi_{n}
$$

for a sequence $\left\{\xi_{n}\right\}$ converging to zero. By Lemma 2.8, we have $u=g x$. Therefore, it follows that

$$
d(g x, S x)=d(u, S x)=d(A, B) .
$$

This completes the proof.

If $\mathrm{g}$ is the identity mapping in Theorem 3.1, then we obtain the following:

Corollary 3.2. Let $X$ be a nonempty set such that $(X, \preceq)$ is a partially ordered set and $(X, d)$ is a complete metric space equipped with the $w$-distance $\mathrm{p}$. Let $\mathrm{A}$ and $\mathrm{B}$ be nonempty closed subsets of $\mathrm{X}$ such that $\mathrm{A}_{0}$ and $\mathrm{B}_{0}$ are nonempty. Assume that the mapping S : A $\rightarrow$ B satisfies the following conditions:

(a) $S$ is a proximally increasing ordered Geraghty's proximal contraction type $(A)$ and (B) with $S\left(A_{0}\right) \subseteq B_{0}$;

(b) there exist $\mathrm{x}_{0}, \mathrm{x}_{1} \in \mathrm{A}_{0}$ with $\mathrm{x}_{0} \preceq \mathrm{x}_{1}$ such that $\mathrm{p}\left(\mathrm{x}_{1}, S \mathrm{x}_{0}\right)=\mathrm{p}(\mathrm{A}, \mathrm{B})$;

(c) if $\left\{x_{n}\right\}$ is an increasing sequence in $A$ converging to $x$, then $x_{n} \preceq x$ for each $n \geqslant 1$ and $\lim _{n \rightarrow \infty} p\left(x, x_{n}\right)=0$.

Then there exists a point $x \in A$ such that $p(x, S x)=p(A, B)$. Moreover, for any $x_{0} \in A_{0}$, the sequence $\left\{x_{n}\right\}$ defined by $p\left(x_{n+1}, S x_{n}\right)=p(A, B)$ converges to the point $x$.

Also, from Theorem 3.1, we have the following:

Corollary $3.3([1])$. Let $X$ be a nonempty set such that $(X, \preceq)$ is a partially ordered set and $(X, d)$ is a complete metric space. Let $\mathrm{A}$ and $\mathrm{B}$ be nonempty closed subsets of $\mathrm{X}$ such that $\mathrm{A}_{0}$ and $\mathrm{B}_{0}$ are nonempty. Assume that the mappings $\mathrm{S}: \mathrm{A} \rightarrow \mathrm{B}$ and $\mathrm{g}: \mathrm{A} \rightarrow \mathrm{A}$ such that $\mathrm{S}$ preserves isometric distance with respect to $\mathrm{g}$ and satisfy the following conditions:

(a) $S$ is a proximally increasing ordered proximal contraction of the first and second kinds with $S\left(A_{0}\right) \subseteq B_{0}$;

(b) $\mathrm{g}$ is a surjective isometry and its inverse is an increasing mapping with $A_{0} \subseteq g\left(A_{0}\right)$; 
(c) there exist $\mathrm{x}_{0}, \mathrm{x}_{1} \in \mathrm{A}_{0}$ with $\mathrm{x}_{0} \preceq \mathrm{x}_{1}$ such that $\mathrm{d}\left(\mathrm{gx_{1 }}, \mathrm{Sx_{0 }}\right)=\mathrm{d}(\mathrm{A}, \mathrm{B})$;

(d) if $\left\{x_{n}\right\}$ is an increasing sequence in $A$ converging to $x$, then $x_{n} \preceq x$ for all $n \geqslant 1$.

Then there exists a point $x \in A$ such that $\mathrm{d}(\mathrm{gx}, \mathrm{Sx})=\mathrm{d}(\mathrm{A}, \mathrm{B})$. Moreover, for any fixed $x_{0} \in \mathrm{A}_{0}$, the sequence $\left\{x_{n}\right\}$ defined by $\mathrm{d}\left(\mathrm{g} \mathrm{x}_{\mathrm{n}+1}, \mathrm{~S} \mathrm{x}_{\mathrm{n}}\right)=\mathrm{d}(\mathrm{A}, \mathrm{B})$ converges to the point $\mathrm{x}$.

Next, we give some examples to illustrate our Theorem 3.1.

Example 3.4. Consider the complete metric space $\mathbb{R}^{2}$ with the metric d defined by

$$
d\left(\left(x_{1}, x_{2}\right),\left(y_{1}, y_{2}\right)\right)=\left|x_{1}-y_{1}\right|+\left|x_{2}-y_{2}\right|
$$

for all $\left(x_{1}, x_{2}\right),\left(y_{1}, y_{2}\right) \in \mathbb{R}^{2}$ and $\left(x_{1}, y_{1}\right) \preceq\left(x_{2}, y_{2}\right)$ if and only if $x_{1} \leqslant x_{2}$ and $y_{1} \leqslant y_{2}$ with the $w$-distance $p$ defined by

$$
p\left(\left(x_{1}, x_{2}\right),\left(y_{1}, y_{2}\right)\right)= \begin{cases}d\left(\left(x_{1}, x_{2}\right),\left(y_{1}, y_{2}\right)\right), & \text { if }\left(x_{1}, x_{2}\right),\left(y_{1}, y_{2}\right) \in F \\ \sqrt{2}, & \text { if }\left(x_{1}, x_{2}\right) \notin F \text { or }\left(y_{1}, y_{2}\right) \notin F\end{cases}
$$

where $F=\{(x, y): x \in\{0,1\},-1 \leqslant y \leqslant 0\}$. Let

$$
A=\{(0, x):-1 \leqslant x \leqslant 0\}, \quad B=\{(1, y):-1 \leqslant y \leqslant 0\} .
$$

Define a mapping $S: A \rightarrow B$ by

$$
S(0, x)=\left(1, \frac{x}{2}\right)
$$

and $g: A \rightarrow A$ is the identity mapping. Then $p(A, B)=1, A_{0}=A, B_{0}=B, \delta(F)=\sqrt{2}$ and $g$ is an isometry.

Now, we prove that $S$ is an ordered Geraghty's proximal contraction type $(A)$ with $\beta \in \mathcal{S}$ defined by

$$
\beta(t)=\frac{1}{1+t}
$$

for all $t>0$. Let $(0, u),(0, v),(0, x),(0, y) \in A$ satisfying

$$
p((0, u), S(0, x))=p(g(0, u), S(0, x))=p(A, B)=1
$$

and

$$
p((0, v), S(0, y))=p(g(0, v), S(0, y))=p(A, B)=1 .
$$

Then $u=\frac{x}{2}$ and $v=\frac{y}{2}$ with $(0, u),(0, v),\left(1, \frac{x}{2}\right),\left(1, \frac{y}{2}\right) \in F$. Moreover, we have

$$
\begin{aligned}
p((0, u),(0, v))=p\left(\left(0, \frac{x}{2}\right),\left(0, \frac{y}{2}\right)\right)=d\left(\left(0, \frac{x}{2}\right),\left(0, \frac{y}{2}\right)\right) & =\left|\frac{x}{2}-\frac{y}{2}\right|=\frac{1}{2}|x-y| \\
& \leqslant \frac{1}{1+|x-y|} \cdot|x-y| \\
& =\beta(p((0, x),(0, y))) p((0, x),(0, y)),
\end{aligned}
$$

which implies that $S$ is an ordered Geraghty's proximal contraction type $(A)$.

Next, we show that $S$ is an ordered Geraghty's proximal contraction type (B). Observe that

$$
\begin{aligned}
p(S(0, u), S(0, v))=p\left(\left(1, \frac{x}{4}\right),\left(1, \frac{y}{4}\right)\right) & =\left|\frac{x}{4}-\frac{y}{4}\right|=\frac{1|x-y|}{2} \\
& \leqslant \frac{1}{1+|x-y|} \cdot \frac{|x-y|}{2} \\
& =\beta(p((0, x),(0, y))) p(S(0, x), S(0, y)) .
\end{aligned}
$$


From the fact that $1+|x-y| \geqslant 1+\frac{|x-y|}{2}$, it follows that

$$
\frac{1}{1+|x-y|} \leqslant \frac{1}{1+\frac{|x-y|}{2}}
$$

and hence

$$
\begin{aligned}
p(S(0, u), S(0, v)) \leqslant \frac{1}{1+|x-y|} \cdot \frac{|x-y|}{2} & \leqslant\left(\frac{1}{\left.1+\frac{|x-y|}{2}\right) \cdot \frac{|x-y|}{2}}\right. \\
& =\beta\left(p\left(\left(0, \frac{x}{2}\right),\left(0, \frac{y}{2}\right)\right)\right) p\left(\left(0, \frac{x}{2}\right),\left(0, \frac{y}{2}\right)\right) \\
& =\beta(p(S(0, x), S(0, y))) p(S(0, x), S(0, y)) .
\end{aligned}
$$

Therefore, $S$ is an ordered Geraghty's proximal contraction type (B). Furthermore, a point $(0,0) \in A$ is such that

$$
p(g(0,0), S(0,0))=p(A, B)=1 .
$$

Next, we present an example which shows the hypotheses in Theorem 3.1 do not guarantee the uniqueness of the best proximity point.

Example 3.5. Let $X=\{(-2,0),(0,-2),(2,0),(0,2)\}$ with the Euclidean metric and the $w$-distance $p=d$. Consider the usual order $\left(x_{1}, y_{1}\right) \preceq\left(x_{2}, y_{2}\right)$ if and only if $x_{1} \leqslant x_{2}$ and $y_{1} \leqslant y_{2}$. Let $A=\{(-2,0),(0,-2)\}$ and $B=\{(2,0),(0,2)\}$. Define a mapping $S: A \rightarrow B$ by

$$
S(x, y)=(-y,-x)
$$

and $g: A \rightarrow A$ is the identity mapping. Then we have

$$
d(A, B)=2 \sqrt{2}, \quad A_{0}=A, \quad B=B, S(A) \subseteq B .
$$

Further, $(-2,0),(0,-2) \in A$ are such that $(-2,0) \preceq(-2,0)$ and $(0,-2) \preceq(0,-2)$. So, it is easy to see that $S$ is an ordered Geraghty's proximal contractions type $(A)$ and $(B)$ with $\beta \in \mathcal{S}$ defined by $\beta(t)=\frac{1}{1+t}$ for any $t>0$. Also, we have

$$
d((-2,0), S(-2,0))=d((-2,0),(0,2))=2 \sqrt{2}=d((0,-2),(2,0))=d((0,-2), S(0,-2)) .
$$

Therefore, $(-2,0)$ and $(0,-2)$ are the best proximity points of the mapping $S$.

Now, we recall the following condition, which is given by Nieto and Rodriguez-Lopez [13], for the uniqueness of the point $x \in A$ satisfying the condition $p(g x, S x)=p(A, B)$.

(B) For any $x, y \in X$, there exists $z \in X$ which is comparable to $x$ and $y$.

Theorem 3.6. Let $X$ be a nonempty set such that $(X, \preceq)$ is a partially ordered set and $(X, d)$ is a complete metric space equipped with the $w$-distance $p$. Let $A$ and $B$ be nonempty closed subsets of $X$ such that $\mathrm{A}_{0}$ and $\mathrm{B}_{0}$ are nonempty with $\mathrm{A}_{0}$ satisfying the condition (B). Assume that the mappings $\mathrm{S}: \mathrm{A} \rightarrow \mathrm{B}$ and $\mathrm{g}: \mathrm{A} \rightarrow \mathrm{A}$ such that $\mathrm{S}$ preserves isometric distance with respect to $\mathrm{g}$ and satisfy the following conditions:

(a) $S$ is a proximally increasing ordered Geraghty's proximal contraction type $(A)$ and (B) with $S\left(A_{0}\right) \subseteq B_{0}$;

(b) $\mathrm{g}$ is a surjective isometry and its inverse is an increasing mapping with $\mathrm{A}_{0} \subseteq \mathrm{g}\left(\mathrm{A}_{0}\right)$; 


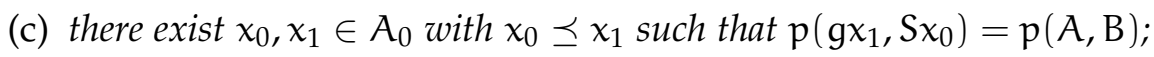

(d) if $\left\{x_{n}\right\}$ is an increasing sequence in $A$ converging to $x$, then $x_{n} \preceq x$ for all $n \geqslant 1$ and $\lim _{n \rightarrow \infty} p\left(x, x_{n}\right)=0$.

Then there exists a unique point $x \in A$ such that $\mathrm{p}(\mathrm{gx}, \mathrm{Sx})=\mathrm{p}(\mathrm{A}, \mathrm{B})$. Moreover, for any $\mathrm{x}_{0} \in \mathrm{A}_{0}$, the sequence $\left\{x_{n}\right\}$ defined by $p\left(g x_{n+1}, S x_{n}\right)=p(A, B)$ converges to the point $x$.

Proof. Now, we show only the uniqueness of the best proximity point of the mapping S. Suppose that there exist $x$ and $x^{*}$ in $A$ such that

$$
p(g x, S x)=p(A, B), \quad p\left(g x^{*}, S x^{*}\right)=p(A, B) .
$$

Let $x$ be comparable to $x^{*}$, that is, $x \preceq x^{*}$ or $x^{*} \preceq x$. Suppose that $x \preceq x^{*}$. Then, since $S$ is an ordered Geraghty's proximal contraction type $(A)$ with the isometry of $g$, we have

$$
p\left(x, x^{*}\right)=p\left(g x, g x^{*}\right) \leqslant \beta\left(p\left(x, x^{*}\right)\right) p\left(x, x^{*}\right) .
$$

If $p\left(x, x^{*}\right) \neq 0$, then, by (3.10), we have

$$
1=\frac{p\left(x, x^{*}\right)}{p\left(x, x^{*}\right)} \leqslant \beta\left(p\left(x, x^{*}\right)\right)<1
$$

which is a contradiction. Thus $p\left(x, x^{*}\right)=0$. If $x \preceq x$, then, by the same method as above, we can see that $p(x, x)=0$. Thus, by Lemma 2.8, we obtain $x=x^{*}$. Similarly, in the case of $x^{*} \preceq x$, we can see that $x=x^{*}$.

Suppose that $x$ is not comparable to $x^{*}$. Since $A_{0}$ satisfies the condition (B), there exists $z \in A_{0}$ such that $z$ is comparable to $x$ and $x^{*}$, that is, $x \preceq z$ or $z \preceq x$ and $x^{*} \preceq z$ or $z \preceq x^{*}$.

If $x \preceq z$ and $x^{*} \preceq z$, since $\left\{x_{n}\right\}$ converges to $x$, then it follows the condition (d) that $x_{n} \preceq x \preceq z$. Since $z \in A_{0}$, from the fact that $S\left(A_{0}\right) \subseteq B_{0}$ and $A_{0} \subseteq g\left(A_{0}\right)$, it follows that there exists a point $v_{0} \in A_{0}$ such that

$$
p\left(g v_{0}, S z\right)=p(A, B) .
$$

Further, we have $g v_{0} \in A_{0}$. Since $S$ is proximally increasing, $g x \preceq g v_{0}, g x^{*} \preceq g v_{0}$, and its inverse is an increasing mapping, we also have $x \preceq v_{0}$ and $x^{*} \preceq v_{0}$. Using the similar fashion, we can find $v_{n}, v_{n+1} \in A_{0}$ such that $x \preceq v_{n}, x^{*} \preceq v_{n}$ and

$$
p\left(g v_{n+1}, S v_{n}\right)=p(A, B) .
$$

By (3.9) and (3.11), $S$ is an ordered Geraghty's proximal contraction type (A) with the isometry of $g$, we have

$$
p\left(x, v_{n+1}\right)=p\left(g x, g v_{n+1}\right) \leqslant \beta\left(p\left(x, v_{n}\right)\right) p\left(x, v_{n}\right) .
$$

If $\lim _{n \rightarrow \infty} p\left(x, v_{n}\right) \neq 0$, then we have

$$
1=\lim _{n \rightarrow \infty} \frac{p\left(x, v_{n+1}\right)}{p\left(x, v_{n}\right)} \leqslant \lim _{n \rightarrow \infty} \beta\left(p\left(x, v_{n}\right)\right)<1,
$$

which is a contradiction, and hence $\lim _{n \rightarrow \infty} p\left(x, v_{n}\right)=0$. Therefore, it follows that $p\left(x, v_{n}\right) \leqslant \alpha_{n}$ for a sequence $\left\{\alpha_{n}\right\}$ converging to zero. Since $p(x, x)=0$, putting $x_{n}:=x, \beta_{n}:=0$ for all $n \geqslant 1$ and applying Lemma 2.8 (2), it follows that $v_{n} \rightarrow x$ as $n \rightarrow \infty$. Similarly, we can prove that $v_{n} \rightarrow x^{*}$ as $n \rightarrow \infty$. By the uniqueness of the limit, we conclude that $x=x^{*}$. For other case, we can prove the same results by the same argument. This completes the proof.

If $g$ is the identity mapping in Theorem 3.6, then we obtain the following: 
Corollary 3.7. Let $X$ be a nonempty set such that $(X, \preceq)$ is a partially ordered set and $(X, d)$ is a complete metric space equipped with the $w$-distance $\mathrm{p}$. Let $\mathrm{A}$ and $\mathrm{B}$ be nonempty closed subsets of $\mathrm{X}$ such that $\mathrm{A}_{0}$ and $\mathrm{B}_{0}$ are nonempty with $\mathrm{A}_{0}$ satisfying the condition (B). Assume that the mapping $\mathrm{S}: \mathrm{A} \rightarrow \mathrm{B}$ satisfies the following conditions:

(a) $S$ is a proximally increasing ordered Geraghty's proximal contraction type $(A)$ and (B) with $S\left(A_{0}\right) \subseteq B_{0}$;

(b) there exist $\mathrm{x}_{0}, \mathrm{x}_{1} \in \mathrm{A}_{0}$ with $\mathrm{x}_{0} \preceq \mathrm{x}_{1}$ such that $\mathrm{p}\left(\mathrm{x}_{1}, S \mathrm{x}_{0}\right)=\mathrm{p}(\mathrm{A}, \mathrm{B})$;

(c) if $\left\{x_{n}\right\}$ is an increasing sequence in $A$ converging to $x$, then $x_{n} \preceq x$ for all $n \geqslant 1$ and $\lim _{n \rightarrow \infty} p\left(x, x_{n}\right)=0$.

Then there exists a unique point $x \in A$ such that $p(x, S x)=p(A, B)$. Moreover, for any $x_{0} \in A_{0}$, the sequence $\left\{x_{n}\right\}$ defined by $p\left(x_{n+1}, S x_{n}\right)=p(A, B)$ converges to the point $x$.

Corollary 3.8. Let $X$ be a nonempty set such that $(X, \preceq)$ is a partially ordered set and $(X, d)$ is a complete metric space. Let $\mathrm{A}$ and $\mathrm{B}$ be nonempty closed subsets of $\mathrm{X}$ such that $\mathrm{A}_{0}$ and $\mathrm{B}_{0}$ are nonempty with $\mathrm{A}_{0}$ satisfying the condition (B). Assume that the mappings $\mathrm{S}: \mathrm{A} \rightarrow \mathrm{B}$ and $\mathrm{g}: \mathrm{A} \rightarrow \mathrm{A}$ such that $\mathrm{S}$ preserves isometric distance with respect to $\mathrm{g}$ and satisfy the following conditions:

(a) $S$ is a proximally increasing ordered proximal contraction of the first and second kinds with $S\left(A_{0}\right) \subseteq B_{0}$;

(b) $g$ is a surjective isometry and its inverse is an increasing mapping with $A_{0} \subseteq g\left(A_{0}\right)$;

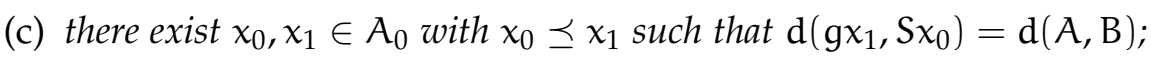

(d) if $\left\{x_{n}\right\}$ is an increasing sequence in $A$ converging to $x$, then $x_{n} \preceq x$ for all $n \geqslant 1$.

Then there exists a point $x \in A$ such that $d(g x, S x)=d(A, B)$. Moreover, for any $x_{0} \in A_{0}$, the sequence $\left\{x_{n}\right\}$ defined by $\mathrm{d}\left(\mathrm{g} \mathrm{x}_{\mathrm{n}+1}, \mathrm{~S} \mathrm{x}_{\mathrm{n}}\right)=\mathrm{d}(\mathrm{A}, \mathrm{B})$ converges to the point $\mathrm{x}$.

Remark 3.9. In this paper, we proved some optimal optimization problems for Geraghty's proximal contractions type $(A)$ and type $(B)$ in the setting of partially ordered sets via the $w$-distance. The main results improve and extend some best proximity point theorems given by Sadiq Basha [1] in partially ordered metric spaces under the ordered proximal contractions of the first and second kind. Finally, we give some examples to illustrate the main results in this paper.

\section{Acknowledgment}

The authors would like to thank the referee for valuable comments and suggestions for the improvement of our manuscript. The first author would like to thank the Thailand Research Fund (Grant No. TRG5880221) for financial support.

\section{References}

[1] S. S. Basha, Discrete optimization in partially ordered sets, J. Global Optim., 54 (2012), 511-517. 1, 2.15, 2.16, 3.3, 3.9

[2] J. Caballero, J. Harjani, K. Sadarangani, A best proximity point theorem for Geraghty-contractions, Fixed Point Theory Appl., 2012 (2012), 9 pages. 1

[3] Y. J. Cho, A. Gupta, E. Karapınar, P. Kumam, W. Sintunavarat, Tripled best proximity point theorem in metric spaces, Math. Inequal. Appl., 16 (2013), 1197-1216. 1

[4] K. Fan, Extensions of two fixed point theorems of F. E. Browder, Math. Z., 112 (1969), 234-240. 1

[5] M. A. Geraghty, On contractive mappings, Proc. Amer. Math. Soc., 40 (1973), 604-608. 1

[6] M. Imdad, F. Rouzkard, Fixed point theorems in ordered metric spaces via w-distances, Fixed Point Theory Appl., 2012 (2012), 17 pages. 1

[7] O. Kada, T. Suzuki, W. Takahashi, Nonconvex minimization theorems and fixed point theorems in complete metric spaces, Math. Japon., 44 (1996), 381-391. 1, 2.8, 2.9

[8] E. Karapinar, Best proximity points of cyclic mappings, Appl. Math. Lett., 25 (2012), 1761-1766. 1

[9] E. Karapınar, W. Sintunavarat, The existence of optimal approximate solution theorems for generalized $\alpha$-proximal contraction non-self mappings and applications, Fixed Point Theory Appl., 2013 (2013), 21 pages.

[10] C. Mongkolkeha, Y. J. Cho, P. Kumam, Best proximity points for Geraghty's proximal contraction mappings, Fixed Point Theory Appl., 2013 (2013), 17 pages. 
[11] C. Mongkolkeha, P. Kumam, Best proximity point theorems for generalized cyclic contractions in ordered metric spaces, J. Optim. Theory Appl., 155 (2012), 215-226.

[12] C. Mongkolkeha, P. Kumam, Some common best proximity points for proximity commuting mappings, Optim. Lett., 7 (2013), 1825-1836. 1

[13] J. J. Nieto, R. Rodríguez-López, Contractive mapping theorems in partially ordered sets and applications to ordinary differential equations, Order, 22 (2005), 223-239. 3

[14] J. B. Prolla, Fixed-point theorems for set-valued mappings and existence of best approximants, Numer. Funct. Anal. Optim., 5 (1983), 449-455. 1

[15] S. Reich, Approximate selections, best approximations, fixed points, and invariant sets, J. Math. Anal. Appl., 62 (1978), 104-113. 1

[16] V. M. Sehgal, S. P. Singh, A generalization to multifunctions of Fan's best approximation theorem, Proc. Amer. Math. Soc., 102 (1988), 534-537. 1

[17] V. M. Sehgal, S. P. Singh, A theorem on best approximations, Numer. Funct. Anal. Optim., 10 (1989), 181-184. 1

[18] N. Shioji, T. Suzuki, W. Takahashi, Contractive mappings, Kannan mappings and metric completeness, Proc. Amer. Math. Soc., 126 (1998), 3117-3124. 1

[19] W. Sintunavarat, P. Kumam, Coupled best proximity point theorem in metric spaces, Fixed Point Theory Appl., 2012 (2012), 16 pages. 1

[20] W. Takahashi, Existence theorems generalizing fixed point theorems for multivalued mappings, Fixed point theory and applications, Marseille, (1989), Pitman Res. Notes Math. Ser., Longman Sci. Tech., Harlow, 252 (1991), 397-406. 1

[21] W. Takahashi, Nonlinear functional analysis, Fixed point theory and its applications, Yokohama Publishers, Yokohama, (2000). 2, 2.8 